\title{
A (des)informação sobre medicamentos: o duplo padrão de conduta das empresas farmacêuticas
}

\author{
(Mis)information on drugs: the double standard \\ practiced by pharmaceutical companies
}

José Augusto C. Barros 1

\footnotetext{
1 Departamento de Medicina Social, Centro de Ciências da Saúde, Universidade Federal de Pernambuco. Cidade Universitária, Recife, PE, 50670-900, Brasil. josebarros@uol.com.br
}

\begin{abstract}
Different factors have been identified as influencing drug prescribers. Some studies emphasize the role played by sources of information available to physicians. Reports have been published on the influence of marketing strategies on these health professionals. Such strategies include advertisements in medical journals, sales representatives, free samples, leaflets, distribution of gifts and prizes, etc. The research reported here aimed to identify information provided by a commonly used Brazilian prescription handbook, the Dicionário de Especialidades Farmacêuticas (DEF), in relation to the 44 most frequently sold pharmaceutical products in Brazil, using as parameters the WHO guidelines for information to be included in informative materials offered to physicians. The information was then compared to that included in the PDR (Physicians' Desk Reference) and USP-DI (Drug Information for the Health Care Professional) used by prescribers in the United States. The results showed lack of data in the Brazilian publication (contraindications, side effects, drug interaction), suggesting lack of reliability in prescription quality and thus in the ultimate utilization of drugs.
\end{abstract}

Key words Drugs; Drug Utilization; Drug Industry; Drug Information

Resumo Diferentes fatores foram identificados como exercendo influência sobre o comportamento dos prescritores de medicamentos. Estudos realizados enfatizam o lugar proeminente ocupado pelas fontes de informação disponíveis para os médicos, muitos deles realçando a influência das estratégias de mercadização. Entre elas, pode-se citar os anúncios em revistas médicas, propagandistas, amostras grátis, folhetos, brindes, etc. A presente pesquisa teve o propósito de detectar as informações presentes no Dicionário de Especialidades Farmacêuticas (DEF) sobre as 44 especialidades farmacêuticas mais vendidas, tendo como parâmetro critérios da OMS para comporem material informativo oferecido aos médicos. Essas informações foram, igualmente, comparadas, para os mesmos produtos, com aquelas presentes em dois manuais de uso habitual nos Estados Unidos: Physicians' Desk Reference (PDR) $e$ Drug Information for the Health Care Professional (USP-DI). Os resultados demonstraram ausência, no manual brasileiro de contraindicações, reações adversas, interações, o que permite desconfiar da qualidade da prescrição. Palavras-chave Medicamentos; Uso de Medicamentos; Indústria Farmacêutica; Informação sobre Medicamentos 


\section{A informação sobre medicamentos para os médicos}

Sob a égide da economia de mercado, a informação disponível sobre os medicamentos, destinada ou não aos prescritores, é produzida $\mathrm{e}$ disseminada, em grande medida, pelos próprios fabricantes. Confirmando a importância que é outorgada à publicidade, é suficiente recordar que os produtores gastam nessa atividade entre $15 \%$ e $25 \%$ do seu faturamento global. Visando objetivos mais comerciais que sanitários, é patente o caráter tendencioso desse tipo de informação, com todas as conseqüências daí advindas (Barros, 1995, 1996).

Tem despertado interesse, já há algum tempo, o estudo dos diferentes fatores que exercem influência sobre a prescrição, destacandose, precisamente, entre eles, as fontes de informação utilizadas pelos médicos. Já foi possível detectar que, entre essas fontes, ocupam lugar de destaque as informações produzidas e veiculadas pela indústria farmacêutica, tendo já se constatado que uma atitude pouco crítica por parte dos médicos frente às atividades promocionais da indústria está associada a uma prescrição de baixa qualidade (Hemminki, 1988; Haaijer-Ruskamp \& Hemminki, 1993).

Os fabricantes de medicamentos lançam mão de meios diretos e indiretos para efetuar a publicidade de seus produtos. Incluem-se, no segundo caso, o financiamento de programas de educação continuada, de associações profissionais ou de revistas médicas, além da produção de material tido como educativo, acrescido do relacionamento com autoridades sanitárias, com políticos e com professores e especialistas famosos (os chamados key-physicians ou opinion-makers) (Hemminki \& Pesonen, 1977).

Entre os instrumentos classificados como diretos destacam-se, entre outros, os anúncios em revistas médicas, os propagandistas, a distribuição de amostras grátis, assim como de revistas e outros materiais impressos, e uma série de atividades tidas como sendo de relações públicas, a exemplo de brindes os mais diversos, o financiamento de recepções ou coquetéis em congressos e patrocínio de viagens.

No que diz respeito aos anúncios inseridos nas revistas médicas, há estudos que evidenciam a existência de uma relação direta entre os medicamentos mais anunciados e a freqüência de sua prescrição (Krupka \& Vener, 1985). Outros estudos têm se dirigido à análise dos diferentes aspectos da publicidade inserida em revistas médicas (Avorn \& Hartley, 1982; Schut, 1982, apud Arnau, 1983; Hevia et al., 1990; Tomson \& Weerasuriya, 1990; Wilkes et al., 1992; Herxheimer et al., 1993; Valpuesta et al., 1993).

No que se refere à importância do papel desempenhado pelos propagandistas, os dados existentes são suficientemente elucidativos. Sabe-se, por exemplo, que sobre esse tipo de profissional recai a maior proporção - mais da metade - dos gastos com publicidade feitos pelas empresas (Mackowiak \& Gagnon, 1985; Anônimo, 1991).

Diversos estudos (alguns já conduzidos nos anos 50 e outros mais recentemente), realizados com o propósito de identificar as fontes de informação e de influência sobre a prescrição, assinalam o lugar proeminente do propagandista como meio através do qual chega ao médico o conhecimento da presença no mercado de um novo produto (Christensen \& Bush, 1981). Observou-se, igualmente, que as características e o nível de formação (tanto a educação geral como a educação científica) dos médicos atuam fazendo com que a informação oral seja mais retida e utilizada que a escrita (Peters, 1981). São igualmente múltiplas as evidências de que o contato pessoal tem uma eficácia maior para conformar o comportamento do prescritor (Harvey et al., 1986; American College of Physicians, Health and Public Policy Committee, 1988).

Conclusões advindas da extensa revisão realizada por Lexchin, na qual se comenta os resultados de uma série de investigações sobre diferentes aspectos da prática dos propagandistas e da relação entretida pela indústria com os mesmos, são taxativas ao concluir que estes profissionais são, sobretudo, agentes de vendas (Hemminki, 1977a, 1977b, 1988; Lexchin, 1989).

Para contrapor-se às fontes de informação classificadas como comerciais, com a má qualidade e tendenciosidade a elas inerentes, existem as chamadas fontes profissionais de informação. Neste grupo, e com um potencial maior ou menor na contribuição pretendida de melhorar a qualidade da informação brindada aos prescritores, podemos incluir: textos de farmacologia e/ou de terapêutica, além de outros livros especializados (como é o caso dos que tratam das reações adversas ou dos que versam sobre o manejo de enfermidades específicas); centros de consulta terapêutica; centros de informação sobre medicamentos dirigidas aos usuários e/ou aos profissionais prescritores; boletins ou outras publicações regulares; formulários de medicamentos ou guias terapêuticos.

Quanto aos prescritores, a disponibilidade de informações de cunho pluridisciplinar, sob 
a responsabilidade de instituições independentes, sem vínculos com os produtores, funciona, certamente, como uma ferramenta indispensável em qualquer programa que se proponha a intervir sobre a demanda. Entre as medidas que devem ser incluídas em um programa deste tipo, caberia ressaltar a elaboração de um Índice Farmacológico ou Guia Terapêutico. Várias recomendações da Organização Mundial da Saúde (OMS) chamam a atenção para a necessidade de que cada país tome a iniciativa de organizar seu formulário terapêutico. Estes teriam o propósito prioritário de racionalizar o consumo, estabelecendo critérios para a utilização dos medicamentos e facilitando, desta forma, as opções do médico. Nesta linha, diferentes países prepararam seu guia, a exemplo dos formulários italianos para a prática extra-hospitalar, vindos à luz nos anos de 1978 e 1981, ou do Index Farmacológico (editado em Catalunha/Espanha, em 1980, e posteriormente reeditado em 1984, 1988 e 1992), cuja preparação se pautou na sistemática adotada pela OMS quando da elaboração da lista de medicamentos essenciais (Arnau, 1983).

Em diferentes países, tal como no Brasil, os supostos guias terapêuticos disponíveis, e que são os mais utilizados como fonte de consulta no cotidiano dos prescritores, na verdade, se constituem em mais um veículo de propaganda, mesmo que tentem se fazer passar como fonte objetiva e confiável de informação. Assim sendo, a avaliação realizada no Compendium of Pharmaceuticals and Specialities (CPS) do Canadá é bastante ilustrativa (Bell \& Osterman, 1983). A análise percuciente realizada concluiu que o CPS listava de maneira acrítica produtos inadequados, ampliava os benefícios e subestimava os efeitos adversos de muitas especialidades farmacêuticas. Adicionalmente, não efetuava comparação da eficácia de fármacos indicados para uma mesma enfermidade nem tão pouco dava orientação a respeito da terapêutica de eleição.

Até agora não existe, no Brasil, um verdadeiro guia terapêutico, embora, um grupo de profissionais vinculados à Escola Nacional de Saúde Publica (Fundação Oswaldo Cruz) tenha tomado a iniciativa pioneira de elaborar um guia terapêutico (Rozenfeld \& Pepe, 1992), publicado por uma editora privada. Entretanto, a divulgação e utilização deste guia é ainda muito limitada. Persiste um predomínio intensivo das fontes de informação de natureza comercial, representado, no estudo realizado, sobretudo, pelo Dicionário de Especialidades Farmacêuticas (DEF), editado e distribuído anualmente sob os auspícios da entidade que con- grega os produtores. Partimos do pressuposto de que o DEF apresentaria deficiências bem mais significativas que aquelas antes mencionadas para o CPS canadense.

Em relação aos boletins sobre medicamentos, implementados nas últimas três décadas, há o empenho de que se mantenham independentes da indústria farmacêutica e, na medida do possível, dos governos. Eles oferecem a seus leitores informações profissionais imparciais e sem distorções. São publicações caracterizadas pelo cunho sintético das revisões críticas que oferecem aos profissionais de saúde e têm como objetivo ajudá-los a tomar as melhores decisões possíveis a respeito de tratamento. $\mathrm{O}$ interesse é oferecer aos leitores um enfoque distinto da maioria dos artigos sobre fármacos publicados em revistas médicas, isto é, os problemas terapêuticos são examinados do ponto de vista do prescritor; fazem-se revisões do papel de um fármaco específico, discutindo as evidências científicas de seu valor prático; comparam-se estratégias terapêuticas e fármacos que competem entre si e são apresentadas conclusões sobre quando e porque, assim como sobre a maneira de utilizar um determinado tratamento, se é que este se justifica. Os boletins se preocupam em explicar as informações essenciais para usar os fármacos. Só que pela natureza da publicação carecem de espaço para fornecer detalhes (este nível de aprofundamento pode encontrar-se nos livros ou nas chamadas data sheet). Neste último caso, entretanto, eles expressam muito mais os pontos de vista dos produtores ou da agência estatal, responsável pelo registro. Em todo caso, estas informações apresentam, com freqüência, empecilhos ao prescritor para traduzi-las à prática clínica (Herxheimer, 1991).

Os estudos orientados à avaliação do impacto dos boletins sobre a prescrição já realizados são muito poucos e, às vezes, apresentam resultados contraditórios. A revisão sobre o tema feita por Plumridge chegou à conclusão de que, mesmo sendo preciso explicitar as razões determinantes do êxito ou fracasso dos boletins terapêuticos, tudo indica que muitos deles não conseguem alcançar sua clientela alvo. (Plumridge \& Berbatis, 1989). A revista francesa Prescrire, da qual existe, igualmente, uma edição internacional, publicada em inglês, é um bom exemplo na direção de tornar tais publicações mais atraentes e acessíveis. 


\section{Procedimentos metodológicos}

Para a consecução dos objetivos propostos na pesquisa realizada a partir das cinqüenta especialidades farmacêuticas mais vendidas no Brasil, foram comparadas as informações existentes no PDR (Physicians' Desk Reference) e USP-DI (Drug Information for the Health Care Professional) dos Estados Unidos com aquelas presentes, para os mesmos produtos, no DEF brasileiro. Os parâmetros adotados para a comparação, foram aqueles propostos pela OMS como correspondendo às informações indispensáveis ao prescritor e, como tal, devendo constar, rotineiramente, de todo tipo de material dirigido a esse profissional. Esses critérios são apresentado a seguir: nome genérico, mecanismo de ação, efeito farmacológico, indicação, contra-indicação, posologia, reações adversas, interações, superdosagem, apresentação, fabricante e importador.

\section{Resultados}

Os dados coletados tiveram que se restringir a 44 das cinqüenta especialidades farmacêuticas mais vendidas no Brasil, em virtude de seis produtos serem à base de dipirona, fármaco que não consta dos textos de referência dos Estados Unidos e que tem sofrido severas restrições, ou mesmo franca restrições, em diferentes países, em virtude de seu potencial de provocar reações adversas no sistema hematopoiético (agranulocitose).

No seu conjunto, a comparação efetuada a partir das informações disponíveis nos três manuais evidenciou discrepâncias importantes, tal como se pode apreender nos resultados apresentados em seguida.

No conjunto das especialidades farmacêuticas estudadas, nos montantes explicitados em cada caso, a proporção de informações ausentes nos três compêndios utilizados é apre- sentada na Tabela 1. A Tabela 2 sintetiza as informações quanto à ausência dos critérios da OMS, considerando os três compêndios estudados.

A título de ilustração, a Tabela 3 evidencia as discrepâncias e coincidências encontradas quanto aos critérios propostos pela OMS, no tocante às informações relacionadas a vinte produtos selecionados na lista dos mais vendidos no Brasil.

\section{Discussão}

Estudos já realizados dão conta da existência de um duplo padrão de conduta das empresas produtoras de medicamentos, conforme o país onde estejam instaladas. Um desses estudos, realizado entre 1987 e 1990 e publicado em 1993, foi elaborado pelo congresso dos Estados Unidos, analisando o conteúdo de bulas e textos presentes em embalagens de produtos similares, perfazendo um total de 243 , oriundos de 19 empresas com sede no mencionado país e presentes no Quênia, Panamá, Tailândia e Brasil. O termo de referência seguido foi o do material que recebera aprovação da FDA (Food and Drug Administration), bem como a literatura especializada. Problemas foram detectados com respeito às indicações (item que apresentou maior número de discrepâncias, mais precisamente, em $26 \%$ dos textos das bulas e das embalagens estudadas), contra-indicações, reações adversas (em um quarto dos casos, elas não eram mencionadas ou não mereceram o devido realce), advertências e precauções (United States Congress, 1993).

A análise dos dados encontrados aponta para deficiências de ordem qualitativa e quantitativa nas informações que estão disponíveis no compêndio brasileiro (DEF), fato que, certamente, contribui para uma prescrição e utilização menos racional de uma série de produtos, entre os mais vendidos no país. E isto é

Tabela 1

Percentagem de critérios sugeridos pela OMS ausentes nos textos de referência em relação ao total de especialidades mais vendidas no Brasil.

\begin{tabular}{lc}
\hline Manual & Proporção (\%) de informações ausentes \\
\hline Physician's Desk Reference (PDR) & 10,8 \\
Drug Information for the Health Care Professional (USP-DI) & 8,0 \\
Dicionário de Especialidades Farmacêuticas (DEF) & 65,2 \\
\hline
\end{tabular}


Tabela 2

Porcentagem de cada critério sugerido pela OMS de acordo com sua ausência nos textos de referência no Brasil e nos Estados Unidos para as 44 especialidades mais vendidas no Brasil (em US\$).

\begin{tabular}{lccc}
\hline Critérios & $\begin{array}{c}\text { PDR } \\
\%\end{array}$ & $\begin{array}{c}\text { Manual } \\
\text { USP-DI } \\
\%\end{array}$ & $\begin{array}{c}\text { DEF } \\
\%\end{array}$ \\
\hline Efeitos adversos & 5,6 & - & 57,2 \\
Posologia & - & - & 37,7 \\
Nome genérico & - & - & - \\
Interações & 38,8 & 2,8 & 41,7 \\
Indicações & 2,3 & 2,8 & 13,8 \\
Contraindicações & 8,3 & - & 30,5 \\
Superdosagem & 11,1 & 69,4 & 38,8 \\
Mecanismo de ação & 13,8 & 5,5 & 55,5 \\
Efeitos farmacológicos & 5,5 & - & 41,6 \\
\hline
\end{tabular}

Tabela 3

Síntese das informações disponíveis em relação às 20 especialidades mais vendidas no Brasil em 1994.

\begin{tabular}{|c|c|c|c|c|c|c|c|c|c|c|c|c|c|c|c|c|c|c|c|c|c|}
\hline \multirow[t]{3}{*}{ Produto } & \multicolumn{21}{|c|}{ Fonte de Informações } \\
\hline & \multicolumn{3}{|c|}{$\begin{array}{c}\text { Efeitos } \\
\text { Adversos }\end{array}$} & \multicolumn{3}{|c|}{ Posologia } & \multicolumn{3}{|c|}{ Interações } & \multicolumn{3}{|c|}{$\begin{array}{c}\text { Contra- } \\
\text { indicações }\end{array}$} & \multicolumn{3}{|c|}{ Superdosagem } & \multicolumn{3}{|c|}{$\begin{array}{l}\text { Mecanismo } \\
\text { de Ação }\end{array}$} & \multicolumn{3}{|c|}{$\begin{array}{c}\text { Efeitos } \\
\text { Farmacológicos }\end{array}$} \\
\hline & PDR & DEF & USP-DI & PDR & DEF & USP-DI & PDR & DEF & USP-DI & PDR & DEF & USP-DI & PDR & DEF & USP-DI & PDR & DEF & USP-DI & PDR & DEF & USP-DI \\
\hline \multicolumn{22}{|l|}{ AAS } \\
\hline \multicolumn{22}{|c|}{ AMPLACILINA } \\
\hline \multicolumn{22}{|l|}{ BIOFENAC } \\
\hline \multicolumn{22}{|l|}{ BUSCOPAN } \\
\hline \multicolumn{22}{|l|}{ CEBION } \\
\hline \multicolumn{22}{|l|}{ CECLOR } \\
\hline \multicolumn{22}{|l|}{ CEFAMOX } \\
\hline \multicolumn{22}{|l|}{ DIPROSPAN } \\
\hline \multicolumn{22}{|l|}{ FELDENE } \\
\hline \multicolumn{22}{|c|}{ FRADEMICINA } \\
\hline \multicolumn{22}{|c|}{ HICONCIL } \\
\hline \multicolumn{22}{|l|}{ HIGROTON } \\
\hline \multicolumn{22}{|l|}{ HYDERGINE } \\
\hline \multicolumn{22}{|l|}{ LOSEC } \\
\hline \multicolumn{22}{|l|}{ LORAX } \\
\hline \multicolumn{22}{|l|}{ MICROVLAR } \\
\hline \multicolumn{22}{|l|}{ PREMARIN } \\
\hline \multicolumn{22}{|l|}{ PROFENID } \\
\hline TEGRETOL & & & & & & & & & & & & & & & & & & & & & \\
\hline TYLENOL & & & & & & & & & & & & & & & & & & & & & \\
\hline
\end{tabular}

AAS = Ácido Acetilsalicilico / AMPLACILINA = Ampicilina / BIOFENAC = Diclofenaco / BUSCOPAN = Hioscina + Metamizol / CEBION = Ácido Ascórbico / CECLOR = Cefaclor / CEFAMOX = Cefadroxil / DIPROSPAN = Betametasona /

FELDENE = Piroxican / FRADEMICINA = Lincomicina $/$ HICONCIL = Amoxicilina / HIGROTON = Clortalidona /

HYDERGINE= Dihidroergocristina / LOSEC = Omeprazol / LORAX = Lorazepam / MICROVLAR= Etinil-Estradiol + Levonorgestrel /

PREMARIN = Estrogenos Conjugados $/$ PROFENID = Ketoprofeno $/$ TEGRETOL = Carbamazepina $/$ TYLENOL= Acetominofen 
ainda mais preocupante, na medida em que já se dispõe de informações suficientes quanto ao lugar de destaque ocupado pelo compêndio mencionado, como fonte de consulta dos médicos brasileiros. Estudo anterior realizado em Recife, entrevistando médicos e estudantes de medicina, detectou que mais da metade destes últimos não têm o hábito de consultar textos de farmacologia, tendo $65 \%$ deles afirmado que consultam, via de regra, o DEF. Este, aliás, foi citado como segunda alternativa, entre as prioridades referidas como fonte influenciadora da prescrição mais recentemente feita. Quanto aos médicos incluídos no novo estudo, quase a metade também afirmou não fazer uso regular de compêndios de farmacologia e/ou terapêutica, tendo $52 \%$ das respostas referido o DEF como guia terapêutico sobre o qual recaiu a preferência dos entrevistados (Barros, 1995).

À guisa de exemplos, vale mencionar o fato de que as contra-indicações, efeitos adversos e interações com outros medicamentos não constam do compêndio brasileiro para o Diclofenaco Potássico (Cataflan $\left.{ }^{\circledR}\right)$, Cefalexina (Keflex $^{\circledR}$ ) e Diclofenaco sódico (Voltaren ${ }^{\circledR}$ ), produtos que ocupam o primeiro, segundo e quarto lugares, respectivamente, entre os mais vendidos. Para o primeiro e quarto desses produtos, o PDR aponta reações adversas que vão desde sintomas gastrointestinais até efeitos mais graves, como é o caso da anafilaxia. Em relação ao Captopril (Capoten ${ }^{\circledR}$ ), quinto mais vendido, o DEF ignora as inúmeras reações adversas que esse fármaco pode vir a provocar e que estão claramente anunciadas no PDR, a exemplo de efeitos sobre o sistema cardiovascular (hipotensão, taquicardia) ou genitourinário (insuficiência renal, proteinúria, poliúria, impotência).

\section{Conclusões}

Nosso estudo permite reforçar conhecimento pré-existente quanto a um padrão duplo de conduta das empresas farmacêuticas, conforme estejam elas atuando em país desenvolvido ou subdesenvolvido.

A exaustiva comparação efetuada no presente estudo quanto ao teor de informes sobre parâmetros considerados fundamentais pela OMS entre dois compêndios de uso regular nos Estados Unidos e um no Brasil, como fonte de consulta por parte dos prescritores de medicamentos, evidenciou discrepâncias significativas, com potencial impacto na prescrição. Tendo como base para o cotejo as cinqüenta especialidades mais vendidas no país em 1994, foi possível constatar a inexistência no manual brasileiro (DEF) de dados imprescindíveis, a respeito, por exemplo de contra-indicações, efeitos indesejáveis, interação, entre outros, e que estavam presentes na quase totalidade dos casos nos compêndios utilizados nos Estados Unidos (PDR e USP-DI)

A importância outorgada ao DEF como fonte de informação e consulta regular pelos prescritores no Brasil é preocupante, sobretudo por duas razões:

1) Não existem disponíveis, no país, alternativas de informação que direta ou indiretamente não tenham vínculos com os produtores;

2) O DEF deixa de incluir dados essenciais para respaldar uma prescrição racional, minimizando usos inadequados e riscos potenciais. Comercializado por uma editora de publicações de natureza médica, o referido compêndio, com freqüência, é distribuído gratuitamente por empresas farmacêuticas. Seu conteúdo é fruto de informações fornecidas aos editores pelas empresas fabricantes, sem antes passar pelo crivo de especialistas em farmacoterapia, dando muito mais a impressão de que se trata de um somatório de bulas, em um caso ou outro, mais completas. Tudo isto nos permite concluir que o texto em tela, eivado como está de tendenciosidades, tem conotação mercadológica, sendo muito mais que um manual informativo isento, de natureza técnico-científica, conforme se propõe a ser. 


\section{Referências}

AMERICAN COLLEGE OF PHYSICIANS, HEALTH AND PUBLIC POLICY COMMITTEE, 1988. Improving medical education in therapeutics. Annals of Internal Medicine, 108:145-147.

ANÔNIMO, 1991. Effectiveness of UK pharma reps. Scrip, 1596:4.

ARNAU, J. M., 1983. La Selecció de Medicaments a Catalunya. L'Index Farmacològic i la Utilització de les Fonts d'Informació sobre Medicaments pels Metges i Farmaceutics. Tesi Doctoral, Barcelona: Universitat Autonoma de Barcelona.

AVORN, J. \& HARTLEY, R., 1982. Scientific versus commercial sources of influence on the prescribing behavior of physicians. American Journal of Medicine, 73:4-8.

BARROS, J. A. C., 1995. A Propaganda de Medicamentos - Atentado à Saúde? São Paulo: Sociedade Brasileira de Vigilância de Medicamentos/Editora Hucitec.

BARROS, J. A. C., 1996. A multiplicação de especialidades no mercado farmacêutico. Instrumento a serviço da saúde? Revista Saúde em Debate, 51:59-63.

BELL, R. W. \& OSTERMAN, J. W., 1983. The compendium of pharmaceuticals and specialties: A critical analysis. International Journal of Health Services, 13:107-118.

CHRISTENSEN, D. B. \& BUSH, J. P., 1981. Drug prescribing: Patterns, problems and proposals. Social Science and Medicine, 15A:343-355.

DICIONÁRIO DE ESPECIALIDADES FARMACÊUTICAS (DEF) 96/97, 1996. Rio de Janeiro: Editora de Publicações Científicas Ltda.

HAAIJER-RUSKAMP, F. M. \& HEMMINKI, E., 1993. Social aspects of drugs use. In: Drug Utilization Studies (M. N. G. Dukes, ed.), WHO Regional Publications, European Series 45, pp. 97-124, Copenhagen: WHO.

HAAYER, F., 1982. Rational prescribing and sources of information. Social Science and Medicine, 16: 2017-2023.

HARVEY, K.; STEWART, R.; HEMMING, M.; NAISMITH, N. \& MOULDS, R. F. W., 1986. Educational antibiotic advertising. Medical Journal of Australia, 145:28-32.

HEMMINKI, E., 1977a. Content analysis of drug detailing by pharmaceutical representatives. Medical Education, 11:210-215.

HEMMINKI, E., 1977b. The function of drug company representatives. Scandinavian Journal of Social Medicine, 5:105-114.

HEMMINKI, E. \& PESONEN, T., 1977. An inquiry into associations between leading physicians and the drug industry. Social Science and Medicine, 11: 501-506.

HEMMINKI, E., 1988. Factors influencing prescribing. In: Psychoactive Drugs: Improving Prescribing Practices (H. Ghodse \& I. Khan, eds.), pp. 22-35, Geneva:WHO.
HERXHEIMER, A., 1991. Drug bulletin as part of the scientific literature.Current Contents, 34:4-9.

HERXHEIMER, A.; LUNDBORG, C. S. \&WESTRHOLM, O. B., 1993. Advertisements for medicines in leading medical journals in 18 countries: A 12-month survey of information content and standards. International Journal of Health Services, 23:161172.

HEVIA, A.; AMAYA, A.; SERRANO, J. S.; ARGUELLES, F. \& VALPUESTA, F. J., 1990. Muestreo de la publicidad de la industria quimicofarmacéutica en revistas pediátricas. Revista de Farmacología Clínica Experimental, 7:99-104.

KRUPKA, L. R. \& VENER, A. M., 1985. Prescription drug advertising: Trends and implications. Social Science and Medicine, 20:191-197.

LEXCHIN, J., 1989. Doctors and detailers: Therapeutic education or pharmaceutical promotion? International Journal of Health Services, 19:663679.

MACKOWIAK, J. I. \& GAGNON, J. P., 1985. Effects of promotion on pharmaceutical demand. Social Science and Medicine, 20:1191-1197.

PETERS, G., 1981. Information and education about drugs. In: Pharmaceutical and Health Policy - International Perspectives on Provision and Control of Medicines (R. Blum, A. Herxheimer, C. Stenzel \& J. Woodcock, eds.), pp. 93-121, London: Croom Helm.

PLUMRIDGE, R. R. J. \& BERBATIS, C. G., 1989. Drug bulletins: Effectiveness in modifying prescribing and methods of improving impact. Annals of Pharmacotherapy, 23:330-334.

ROZENFELD, S. \& PEPE, V. L. E., 1992. Guia Terapêutico Ambulatorial. Porto Alegre: Artes Médicas/ ABRASCO.

SCHULTE-SASSE, H. \& HARTOG, R., 1988. La Industria Farmacéutica Alemana en América Latina. Bielefeld: BUKO Pharma-Kampagne.

TOMSON, G. \& WEERASURIYA, K., 1990. 'Codes' and practice: Information in drug advertisements An example from Sri Lanka. Social Science and Medicine, 31:737-741.

UNITED STATES CONGRESS, 1993. Drug Labeling in Developing Countries. Washington, D. C.: Office of Technology Assessment, Government Printing Office.

VALPUESTA, F. J. L.; ALONSO, A. H.; DIAZ, J. A. V.; LOPEZ, J. A. F. \& ALONSO, A. F., 1993. Análisis de la publicidad medicamentosa en revistas médicas no especializadas. Revista Clínica Española, 192:130-133.

WILKES, M. S.; DOBLIN, B. H. \& SHAPIRO, M. F., 1992. Pharmaceutical advertisements in leading medical journal: Experts' assessments. Annals of Internal Medicine, 116:912-919. 